\title{
Exploiting Excited-State Aromaticity To Design Highly Stable Singlet Fission Materials
}

\author{
Kealan J. Fallon, ${ }^{\dagger, \square \odot ~ P e t e r ~ B u d d e n, ~}{ }^{\ddagger} \square$ Enrico Salvadori, ${ }^{\S, \|}$ Alex M. Ganose, ${ }^{\perp, \#, \text { II }}$
}

Christopher N. Savory, ${ }^{\perp, \#}{ }^{\circledR}$ Lissa Eyre, ${ }^{\ddagger}$ Simon Dowland, ${ }^{\ddagger}$ Qianxiang Ai, ${ }^{\nabla}$ Stephen Goodlett, ${ }^{\nabla}$ Chad Risko, ${ }^{\circ}$ David O. Scanlon, ${ }^{\perp, \#, \Psi_{\odot}}$ Christopher W. M. Kay, ${ }^{\|, \otimes}$ Akshay Rao, $^{\ddagger \odot}$

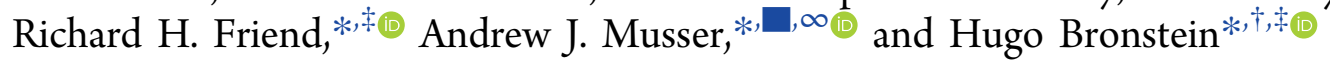

${ }^{\dagger}$ Department of Chemistry, University of Cambridge, Cambridge CB2 1EW, U.K.

${ }^{\ddagger}$ Cavendish Laboratory, University of Cambridge, Cambridge CB3 OHE, U.K.

${ }^{\S}$ Department of Chemistry, University of Turin, Via Pietro Giuria 7, 10125 Torino, Italy

"London Centre for Nanotechnology, University College London, 17-19 Gordon Street, London WC1H 0AH, U.K.

${ }^{\perp}$ Kathleen Lonsdale Materials Chemistry, Department of Chemistry, University College London, 20 Gordon Street, London WC1H OAJ, U.K.

${ }^{\#}$ Thomas Young Centre, University College London, Gower Street, London WC1E 6BT, U.K.

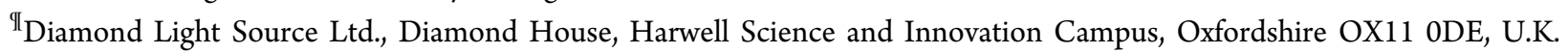

${ }^{\nabla}$ Department of Chemistry and Center for Applied Energy Research, University of Kentucky, Lexington, Kentucky 40506, United States

${ }^{\otimes}$ Department of Chemistry, University of Saarland, 66123 Saarbrücken, Germany

Department of Physics and Astronomy, University of Sheffield, Hicks Building, Hounsfield Road, Sheffield S3 7RH, U.K.

\section{Supporting Information}

\begin{abstract}
Singlet fission, the process of forming two triplet excitons from one singlet exciton, is a characteristic reserved for only a handful of organic molecules due to the atypical energetic requirement for low energy excited triplet states. The predominant strategy for achieving such a trait is by increasing ground state diradical character; however, this greatly reduces ambient stability. Herein, we exploit Baird's rule of excited state aromaticity to manipulate the singlet-triplet energy gap and create novel singlet fission candidates. We achieve this through the inclusion of a [4n] 5-membered heterocycle, whose electronic resonance promotes aromaticity in the triplet state, stabilizing its energy relative to the singlet excited

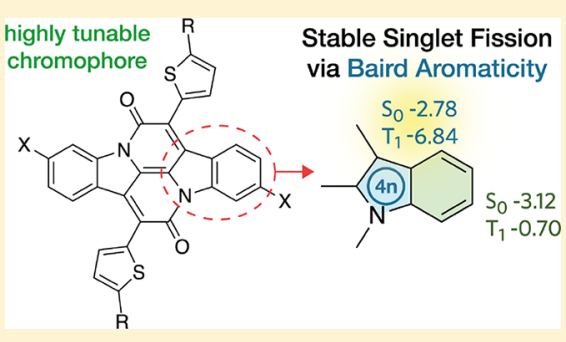
state. Using this theory, we design a family of derivatives of indolonaphthyridine thiophene (INDT) with highly tunable excited state energies. Not only do we access novel singlet fission materials, they also exhibit excellent ambient stability, imparted due to the delocalized nature of the triplet excited state. Spin-coated films retained up to $85 \%$ activity after several weeks of exposure to oxygen and light, while analogous films of TIPS-pentacene showed full degradation after 4 days, showcasing the excellent stability of this class of singlet fission scaffold. Extension of our theoretical analysis to almost ten thousand candidates reveals an unprecedented degree of tunability and several thousand potential fission-capable candidates, while clearly demonstrating the relationship between triplet aromaticity and singlet-triplet energy gap, confirming this novel strategy for manipulating the exchange energy in organic materials.
\end{abstract}

\section{INTRODUCTION}

Phenomena that permit the generation of multiple excitons from the absorption of one photon have received large attention recently due to their potential in photovoltaic cell efficiency enhancement. One such process is singlet fission, whereby two low-energy triplet excited states are generated from one high-energy singlet excited state, allowing quantum efficiencies up to $200 \%$ and offering the potential to surpass the single junction limit in solar cells. ${ }^{1-8}$ Recent advancements in singlet fission have been materials-limited due to the rarity of molecules that meet the essential energetic requirement for the process, namely, that the energy of the lowest triplet excited state $\mathrm{E}\left(\mathrm{T}_{1}\right)$ be on the order of half the energy of the lowest singlet excited state $E\left(S_{1}\right)$. Known systems include rylene dyes, $^{9-12}$ polyenes, ${ }^{13-15}$ donor-acceptor polymers, ${ }^{16-18}$ and derivatives of the linear acenes, particularly tetracene and pentacene, which have become the focus of the field. ${ }^{2,19-31}$ The low triplet energies of the acenes are a function of their migratory Clar's sextets resulting in their large localized

Received: June 14, 2019

Published: August 5, 2019 

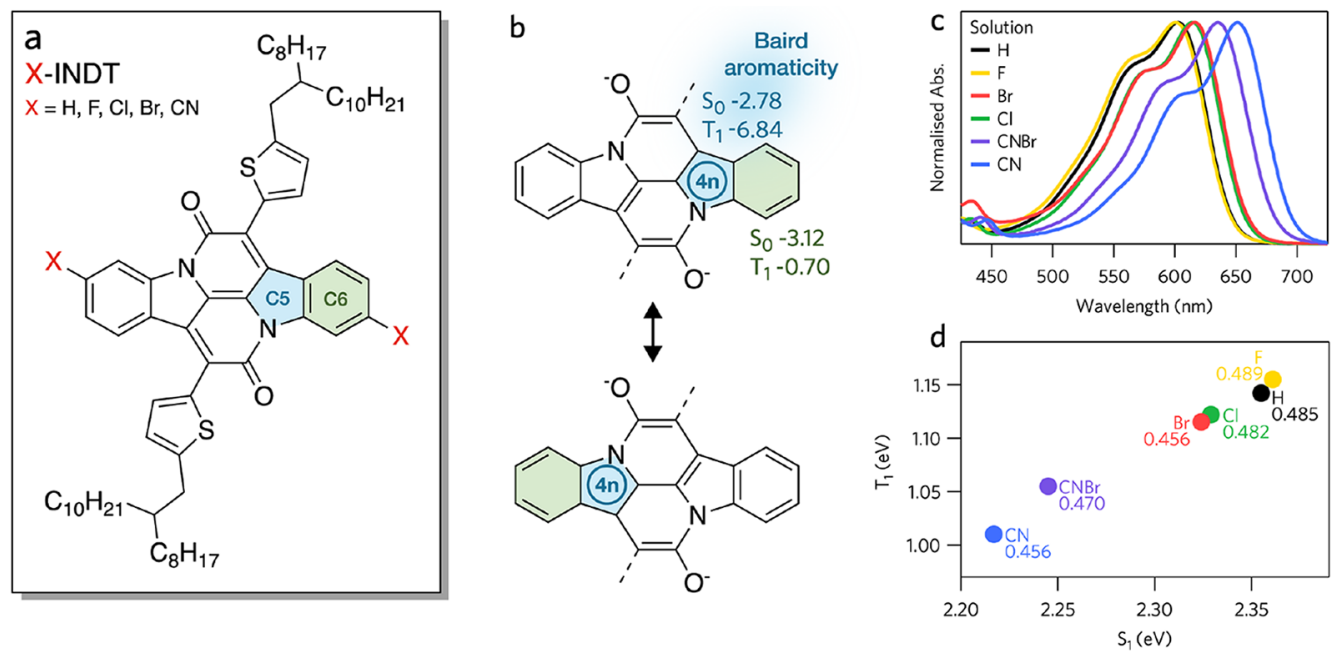

Figure 1. Optical properties and characteristics. (a) The polycyclic aromatic hydrocarbon indolonaphthyridine thiophene (INDT) with labeled rings of interest. (b) Resonance forms of INDT showing origin of Baird [4n] ring (C5, blue). Values of NICS(1) calculations for CN-INDT are shown for $S_{0}$ and $T_{1}$, where a more negative value denotes greater aromaticity. (c) Solution (chloroform) steady-state absorbance spectra of the investigated family of materials. (d) Theoretical energies (eV, TD-DFT/TDA, B3LYP/6-311++G**) of $S_{1}$ and $T_{1}$ relative to the ground state. The ratio of these energies is given inset.

diradical character, which compromises their chemical stability (e.g., pentacene must be stored in the dark under inert gas lest it undergo photocatalyzed dimerization ${ }^{32}$ or oxidation ${ }^{33}$ ). Unfortunately, current established rationales behind synthesizing organic molecules with large singlet $\left(\mathrm{S}_{1}\right)$-triplet $\left(\mathrm{T}_{1}\right)$ energy gaps-such as inducing biradicaloid character-offer the synthetic chemist little assistance in identifying or developing new chromophores for this purpose, although these rules are of very good use to check whether a structure is likely to fulfill the energetic criteria for singlet fission. ${ }^{34-36}$ Merely focusing on increasing the amount of biradical character can often involve cost to chemical stability as it can result in highly reactive localized unpaired electrons, although this can potentially be addressed with careful protection of the radical sites. ${ }^{37,38}$ There is therefore a need for a simple design approach to identify or design materials that undergo singlet fission at no expense to stability.

In the ground state, Hückel's Rule rationalizes the enhanced stability of cyclic systems with $[4 n+2]$ electrons and this concept of aromaticity is a fundamental pillar of chemistry. Lesser known is Baird's Rule, which states that in the triplet state, cyclic systems with $[4 n]$ electrons are aromatic and hence stabilized. ${ }^{39,40}$ Following confirmatory modern theoretical analysis, ${ }^{41-43}$ the rule has been explored in atypical conjugated systems in the past few years. ${ }^{44-47}$ Here, we demonstrate the synthesis and characterization of highly stable, tunable organic materials that undergo singlet fission through exploitation of Baird's aromaticity of the triplet excited state.

\section{RESULTS AND DISCUSSION}

We focus in particular on derivatives of indolonaphthyridine benzene or Cibalackrot (SI Section S1), ${ }^{48}$ previously proposed as a singlet fission candidate. ${ }^{49}$ Quantum chemical calculations (B3LYP/6-311G**) reveal Cibalackrot to possess a low-lying triplet state with $E\left(\mathrm{~T}_{1}\right) / E\left(\mathrm{~S}_{1}\right) \sim 0.5$, affirming its potential for singlet fission. This stabilized triplet state has been previously explained through the stabilized diradical nature of the indigo core. $^{49-51}$ It can likewise be rationalized using Baird aromaticity, due to the presence of $[4 n]$ contribution in its resonance structure (Figure $1 \mathrm{~b}$ and SI Section S2). We have designed a family of materials (Figure 1a) to investigate: (a) whether the indolonaphthyridine chromophore does indeed undergo singlet fission; (b) whether the optical properties and singlet fission ability could be tuned by chemical functionalization; and (c) whether contributions from an aromatic triplet state enhance chemical stability. The full synthesis and characterization of these materials is given in the SI Section $\mathrm{S} 1$, hereafter referred to as $x$-INDT where $x$ is the atom or functional group installed at the $6,6^{\prime}$-positions of the INDT C6 ring (see Figure 1a).

The solution steady-state absorbance spectra (Figure 1c) demonstrate the optical tunability of this family. H-INDT and F-INDT display comparable absorbance with $\lambda_{\max }$ of $602 \mathrm{~nm}$. Both Cl-INDT and Br-INDT exhibit bathochromically shifted but practically identical absorbance with $\lambda_{\max }$ of $615 \mathrm{~nm}$. The addition of strongly electron withdrawing carbonitrile functional groups was achieved through a Rosenmund-von Braun reaction of Br-INDT and furnished both the asymmetric mononitrile/monobromo compound CNBr-INDT and dinitrile compound CN-INDT. The addition of each carbonitrile group narrows the optical band gap of the material significantly, yielding $\lambda_{\max }$ of 635 for the mono- and $\lambda_{\max }$ of $650 \mathrm{~nm}$ for the dicarbonitrile compound.

The energies of the $S_{1}$ and $T_{1}$ states of these materials were calculated using time-dependent density functional theory (TD-DFT) on DFT-optimized structures. These calculations reproduce the observed tunability of the optical energy gap and suggest that all of the molecules possess the relationship of $2 \mathrm{~T}_{1}$ $<S_{1}$ required for singlet fission (Figure 1d). Moreover, the ratio of calculated $T_{1} / S_{1}$ energies reveals that this parameter, and thus suitability for singlet fission, can be tuned through chemical substitution. We do note that the use of TD-DFT for the prediction of triplet state energies is problematic. ${ }^{52}$ However, whether the predicted values for the $\left(T_{1} / S_{1}\right)$ ratio may be over- or underestimated as a whole, the key trendstunability of the ratio and its relation to triplet-state aromaticity-should remain robust. Indeed, these values are 
best taken as a guideline, since incorporation into the solid state inevitably alters the energetic structure to some degree.

We assessed the relative aromaticity of the molecular structures through a variety of computational methods. The aromaticity of the five-membered (C5) ring in the triplet state was assessed using nucleus-independent chemical shift calculations (NICS(1)); this data is given in full in the SI Section S3 and the data for CN-INDT is shown in Figure $1 \mathrm{~b}$. Importantly, the NICS calculations demonstrate how the aromaticity in the $\mathrm{C5}$ ring is significantly increased in the triplet state in accordance with Baird's rules.

Figure 2 shows ACID (anisotropy of the induced current density) plots for the F-INDT in the $S_{0}$ and $T_{1}$ states (see SI

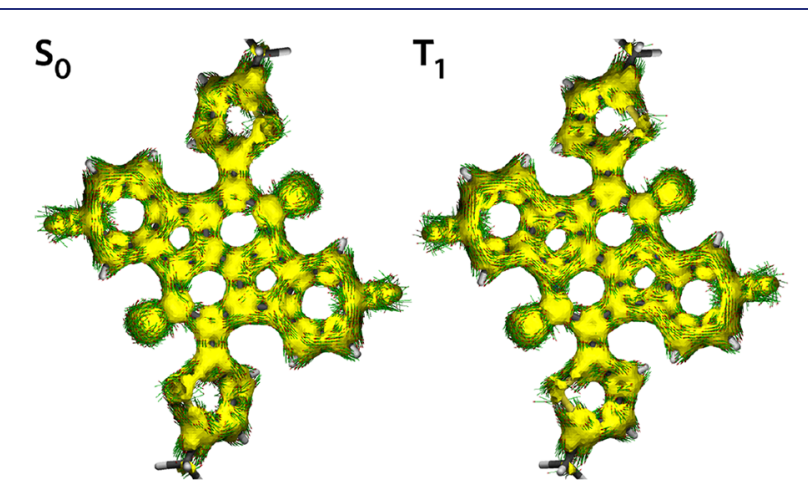

Figure 2. ACID plots for F-INDT in the $S_{0}$ (left) and $T_{1}$ (right) states. The isovalue of the isosurface was set to 0.05 . The induced current density vectors are denoted by the arrows with the length limited to $0.3-1.5$. Larger images available in SI.

for further details). ${ }^{53,54}$ For $S_{0}$, the current density vectors on the outer benzene rings at the ends of backbone show clear diamagnetic features. Such clear features are not present in the other rings, where relatively weak induced currents seem to be more paramagnetic. There is also a weak paramagnetic current running along the periphery of the whole backbone. For $\mathrm{T}_{1}$, the current density vectors on the aforementioned benzene rings couple with the adjacent 5-membered rings, yielding a diamagnetic current restricted to the periphery of the two fused rings. Notice that there is a weak response reflected by small current density vectors that could come from the delocalized $\sigma$-electrons rather than from $\pi$-system. The ACID plots for all molecules are shown in Figure S3.4 with patterns similar to that of F-INDT. Further, NICS- $x y$ and HOMA calculations (see SI) also support an increase in aromatic character of the $\mathrm{C} 5$ ring in the triplet state relative to the ground state.

We focus below on solid-state properties of Br-INDT and CN-INDT, but full characterization of all derivatives is presented in the SI Section S4. In thin film (Figure 3a), all materials exhibit broader absorption and hypsochromically shifted $\lambda_{\text {max }}$ over 500-550 nm. These shifts indicate significant intermolecular interactions between chromophores, here consistent with predominantly H-type exciton coupling. ${ }^{55}$ Large planar molecules such as these exhibit a propensity to aggregate through strong $\pi-\pi$ stacking. The large branched alkyl side chains attached to the out-of-plane thiophene moieties suppress this coupling to a degree and minimize the stabilization of $S_{1}$ in the solid state, which can unfavorably disrupt the energetic balance for singlet fission. ${ }^{49,51}$ Despite the broad and variable line shapes, we note that the thin-film absorption onsets display similar trends to the solution measurements; however, we remind the reader that direct comparison of theoretical (gas phase) energetics to solid state experimental results is unwise as each molecule will experience different intermolecular coupling.

Evidence for Singlet Fission. We investigated the ability of INDT films to undergo singlet fission using time-resolved optical and spin-resonance techniques. In transient absorption spectroscopy, the sample is excited with a short laser pulse and then interrogated with a broadband probe pulse at a controllable time delay (100 fs to $1 \mathrm{~ms})$. Changes in the transmission of the probe reflect the depopulation of the ground state, the absorption spectrum of photoexcited states, and stimulated emission from allowed optical transitions. Full details of the experimental setup are presented in the SI Section S4. Representative transient absorption spectra are shown in Figure $3 \mathrm{~b}$ for Br-INDT and CN-INDT acquired using a ns $-\mu$ s system $\left(\lambda_{\text {exc }}=532 \mathrm{~nm}, \sim 1 \mathrm{~ns}\right)$. A broader probe spectral range and equivalent measurements for the remaining molecules are presented in the SI Section S4.

As shown in Figure 3, we observe a distinct photoinduced absorption band at $\sim 680 \mathrm{~nm}$ (Br-INDT) or $\sim 740 \mathrm{~nm}$ (CNINDT). This species, which persists out to the $\mu$ s time scale with little spectral evolution, should therefore only originate from charges or triplet excitons. Triplet sensitization in solution (green dashed) ${ }^{14}$ yields a similar spectral signature, after taking into consideration the difference in underlying ground-state bleach and a slight bathochromic shift of the $T_{1}$ $\rightarrow \mathrm{T}_{n}$ transition in the solid state which is consistent with other organic systems. ${ }^{14,15,56}$ Thus, we assign the $\mu$ s excited-state absorption to triplet excitons. This is further confirmed via electron paramagnetic resonance (EPR), discussed below. We detect similar evolution in Cl-INDT and CNBr-INDT, while in F-INDT the spectral signature we attribute to triplets decays faster, with a lifetime of $300 \mathrm{~ns}$. In contrast, we do not find evidence for triplets in H-INDT. The primary signature we observe in H-INDT (Figure S4.3) in this temporal regime is a set of positive peaks that match the ground-state absorption and can be assigned to bleaching of the ground state. These are accompanied by a weak photoinduced absorption $(\Delta T / T<0)$ from $650 \mathrm{~nm}$ to the near-infrared which decays on an instrument-limited time scale and is assigned to the singlet. There is no triplet-like absorption in this film, and there are evidently no significant long-lived electronic states in this film. The positive features that outlive the photoinduced absorption are due to thermal modulation of the absorption and reflectivity and are commonly observed in measurements in the ground-state absorption region. ${ }^{11,37,57,58}$

These measurements provide clear evidence of sub-ns triplet formation, but it is difficult to distinguish the mechanismintersystem crossing or singlet fission-from optical spectroscopy alone. We therefore characterized the sublevel populations of the triplet states using time-resolved EPR, which is a powerful tool for probing singlet fission materials. ${ }^{59-63}$ In frozen dilute solution, singlet fissionbeing an intermolecular process-is inactive, and the zero-field sublevel populations of triplet states in these conditions are attributed to intersystem crossing. The frozen solution EPR spectra (Figure 3c) of Br- and CN-INDT exhibit emission/ absorption polarization patterns EEEAAA. In thin film, singlet fission becomes possible and may out-compete intersystem crossing. In such a case, the presence of a new channel for triplet formation would manifest as changes in the triplet 

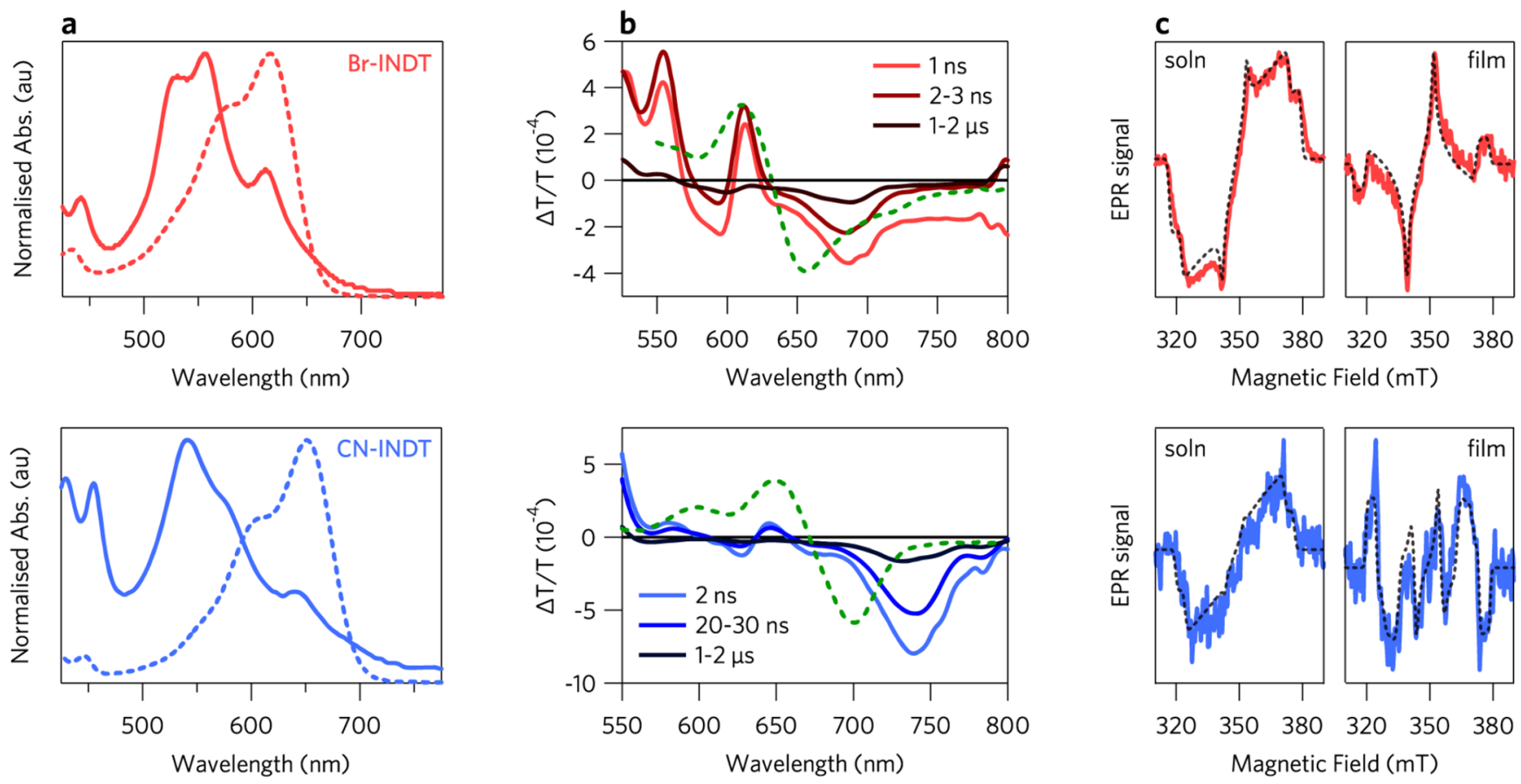

Figure 3. Long-lived triplet excitations in INDT films. (Top, red) Br-INDT, (Bottom, blue) CN-INDT. (a) Solution (dashed line, chloroform) and thin-film (solid line, spun from $5 \mathrm{mg} / \mathrm{mL}$ solutions in chloroform) steady-state absorption spectra. (b) Thin-film transient absorption spectra acquired on ns $-\mu$ s time scale following $1 \mathrm{~ns}$ excitation pulses at $532 \mathrm{~nm}$. Corresponding decay kinetics and spectra acquired over a broader probe range are presented in Figure S4.4. Solution sensitized triplet signatures shown as green dashed line. (c) Experimental (colored) and simulated (black dash) TR-EPR spectra obtained at $10 \mathrm{~K}$ in dilute solution (left) and drop-cast films (right). Simulated parameters for Br-INDT solution ( $D$ $\left.=880 \mathrm{MHz},|E|=185 \mathrm{MHz}, \mathrm{P}_{X}: \mathrm{P}_{Y}: \mathrm{P}_{Z}=0.0: 0.5: 0.5\right)$, Br-INDT film $\left(D=910 \mathrm{MHz},|E|=185 \mathrm{MHz}, \mathrm{P}_{X}: \mathrm{P}_{Y}: \mathrm{P}_{Z}=0.2: 0.6: 0.2\right)$, CN-INDT solution $(D$ $\left.=+830 \mathrm{MHz},|E|=180 \mathrm{MHz}, \mathrm{P}_{X}: \mathrm{P}_{Y}: \mathrm{P}_{Z}=0.0: 0.4: 0.6\right)$. The reported simulation for CN-INDT film is the sum of two components A and $\mathrm{B}$ where the $T_{0}$ sublevel is preferentially populated in high-field (component A: $D=+830 \mathrm{MHz},|E|=180 \mathrm{MHz}$; component $\mathrm{B}: D=-600 \mathrm{MHz},|E|=60$ $\mathrm{MHz}$, see SI Section $\mathrm{S} 4$ for individual simulations). $A$ = enhanced absorption, $E=$ emission.

sublevel populations. The polarization pattern of Br-INDT films is EAEAEA, which cannot be caused by the same intersystem crossing pathway active in dilute solution. The absence of other potential mechanisms allows us to attribute triplet formation in these films to singlet fission. The spectrum of CN-INDT films is more complex- at least two components with different zero-field splitting parameters are present. One component has zero-field splitting parameters similar to those observed in solution but with AEEAAE polarization (SI Section S5). Importantly, this polarization pattern, previously observed in TIPS-tetracene and pentacene dimers, ${ }^{59,60}$ is a signature of triplet pairs formed via singlet fission. ${ }^{59}$ On the basis of this direct evidence of fission in Br-INDT and CN-INDT and the similar sub-ns triplet formation observed in Figure. $3 \mathrm{~b}$, we conclude that singlet fission is active in all of the reported INDT derivatives except H-INDT.

Rapid Triplet Formation. For further insight into the mechanism of singlet fission, we examine the initial decay of $S_{1}$ in Br-INDT and CN-INDT using fs-ps transient absorption spectroscopy $\left(\lambda_{\text {exc }}=530 \mathrm{~nm}, \sim 250 \mathrm{fs}, 37 \mathrm{kHz}\right.$ repetition rate). Representative transient absorption spectra at a high excitation density are shown in Figure $4 \mathrm{a}$ and $4 \mathrm{~b}$ for the same molecules presented in Figure 3. At early times there is a broad photoinduced absorption $(\Delta T / T<0)$ spectrally overlapped with the ground state absorption (making the spectra highly congested in this region) and extending to a peak in the nearinfrared. From comparison of the steady-state absorption and photoluminescence spectra, we can attribute much of the complex structure of this spectrum to overlapping $\Delta T / T>0$ signatures from bleaching of the ground-state absorption and stimulated emission from $S_{1}$.

As the strong initial photoinduced absorption decays, the underlying triplet signal is revealed (685 nm in Br-INDT, 745 $\mathrm{nm}$ in CN-INDT), corresponding well with the ns TA experiment (Figure $3 \mathrm{~b}$ ). Due to the overlapping signals, it is not possible to definitively distinguish the formation time of this signal; however, it is certainly present by $2-3$ ps in $\mathrm{CN}$ INDT, effectively ruling out the formation of these triplets from ISC, and confirming the result from EPR that triplets are originating from singlet fission.

We observe that the dynamics of the loss of the initial photoinduced absorption peaked at $850 \mathrm{~nm}$ are strongly dependent on excitation density. In Br-INDT, at high excitation densities comparable with those required to measure ultrafast photoluminescence ${ }^{64}$ (Figure $4 \mathrm{c}$ ), the decay is $\sim 7$ ps. This rapid decay is likely due to singlet-singlet annihilation. At the lowest excitation densities at which TA was measured, annihilation is not significant, and the decay (biexponential: 58 and $990 \mathrm{ps}$ ) is in agreement with the intrinsic $1 \mathrm{~ns}$ lifetime from time-correlated single-photon counting (Figure 4c). This agreement with photoluminescence measurements in both high- and low-annihilation regimes firmly establishes the initially decaying spectral features, peaked at $850 \mathrm{~nm}$, as signatures of $S_{1}$ and indicates that the INDTs are similarly sensitive to singlet-singlet annihilation as perylenediimides. ${ }^{11}$

Given that the $S_{1}$ lifetime is so strongly affected by singletsinglet annihilation, we would expect that the triplet yield and formation kinetics are also power dependent. Curiously, when 

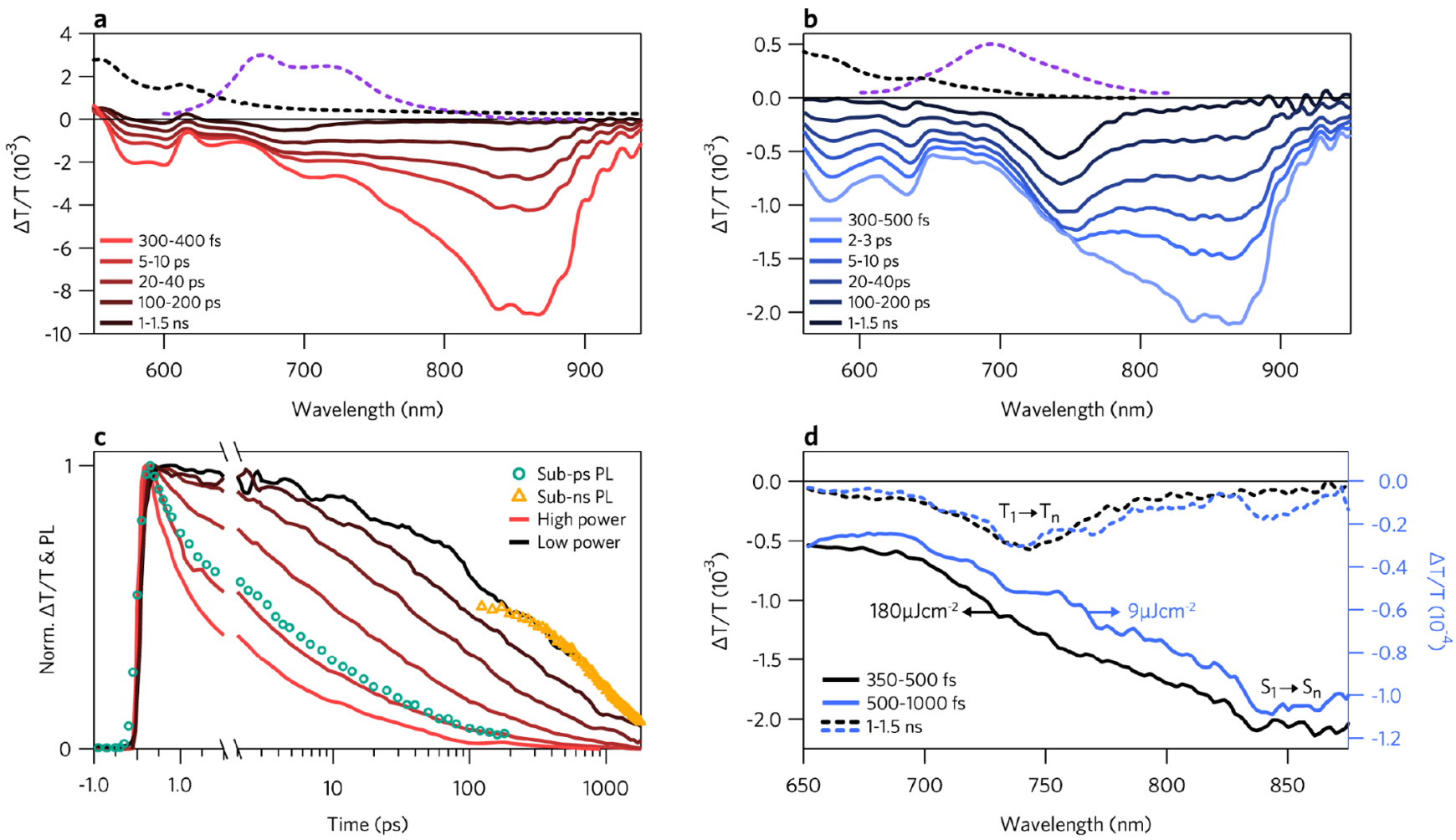

Figure 4. Fast triplet formation in INDT films. Thin-film TA spectra acquired on fs-ps time scale following $250 \mathrm{fs}$ excitation of (a) Br-INDT and (b) CN-INDT. Corresponding decay kinetics and spectra acquired over a broader probe range are presented in Figure S4.4. Dashed spectra show steady-state absorption (black) and PL (purple). (c) Br-INDT excitation-density-dependent TA kinetics (lines) tracking $\mathrm{S}_{1}$ features (860-880 $\mathrm{nm})$, compared with transient grating $\mathrm{PL}^{64}$ kinetics acquired at high laser power $\left(255 \mu \mathrm{J} \mathrm{cm}^{-2}\right.$ per pulse, green circles), and time-correlated singlephoton counting kinetics acquired at low laser power $\left(2 \mathrm{~nJ} \mathrm{~cm}^{-2}\right.$ per pulse, yellow triangles). Note the instrument response of the sub-ns PL measurement is $\sim 300$ ps. (d) Comparison of initial TA signal, taken at the peak in time of the $\mathrm{S}_{1}$ PIA at $850 \mathrm{~nm}$, with the triplet signal at 1-1.5 ns for two different powers: $180 \mu \mathrm{J} \mathrm{cm}$ per pulse (black, left axis) and $9 \mu \mathrm{J} \mathrm{cm}^{-2}$ per pulse (blue, right axis), demonstrating that excitation density does not affect triplet yield in CN-INDT.

we calculate the yield of triplets at $1 \mathrm{~ns}$ in CN-INDT, based on the solution cross sections of the $\mathrm{S}_{1} \rightarrow \mathrm{S}_{n}$ and $\mathrm{T}_{1} \rightarrow \mathrm{T}_{n}$ transitions, it is approximately $60 \%$ at all excitation densities (details of calculations in SI Section S4.9). Triplet yield is not strongly affected by excitation density, despite the clear excitation density dependence of the $S_{1}$ lifetime. This is clearly shown by Figure $4 d$, where the ratio of the initial $S_{1}$ signal to 1 ns $\mathrm{T}_{1}$ signal is unchanged from $9 \mu \mathrm{J} \mathrm{cm}^{-2}$ per pulse to $180 \mu \mathrm{J}$ $\mathrm{cm}^{-2}$ per pulse, while the initial $S_{1}$ decay lifetime falls from $\sim 200$ to 7 ps. On the basis of this evidence, we propose a branching on very early time scales between populations capable and incapable of singlet fission. This is likely due to morphology, with a subset of "hot spots" where triplets can be formed rapidly via singlet fission, ${ }^{24}$ and the resulting triplets are much less susceptible to annihilation due to their lower mobility. Importantly, singlet fission at hotspots in this system is completely outcompeting the singlet annihilation events that occur from Förster transfer on a ps time scale, and singlet fission is therefore likely to be occurring on fs time scales. Meanwhile, a parallel population of singlets formed too far from the hot spots cannot undergo singlet fission, but are mobile enough to show a strong excitation density dependence due to singlet-singlet annihilation. Indeed, preliminary measurements using different solvents or film-forming conditions reveal considerable changes in photoluminescence quantum yield (PLQY), suggesting the density of hot spots can be tuned through processing (see SI Section S4.12). PLQY is measured in steady state at low excitation density, such that the changes in yield cannot be attributed simply to changes in singlet-singlet annihilation channels, but to the rate of intrinsic decay, which we attribute to change in density of singlet fission hotspots. ${ }^{65}$ Given that singlet fission appears to be so fast, we see no reason morphology could not be tuned to produce a quantitative singlet fission yield as has been demonstrated in other systems such as rylene diimides and DPP. ${ }^{9,12,66}$ To capture important trends between the different $x$-INDT in the branching of this mechanism, we compare the magnitude of triplet signature at $\sim 500$ ns normalized by the initial singlet excitation density taken from the excitation pulse energy and the steady state absorption, and using the solution sensitization data to account for differences in triplet absorption cross-section relative to ground state bleach (see SI Section S4.8). This gives relative triplet yields of $\mathrm{H}<\mathrm{F}<$ $\mathrm{CNBr} \approx \mathrm{Cl}<\mathrm{Br}<\mathrm{CN}$, which broadly follows a trend of increasing yield with increasing exothermicity of singlet fission. There is also a possible connection to the strength of intermolecular coupling, ${ }^{27}$ as we observe that the CN-INDT film absorption is most blue-shifted from the solution spectrum with a suppressed 0-0 transition (characteristic of $\mathrm{H}$ aggregation), while that of $\mathbf{H}$-INDT is the least shifted (Figure $3 \mathrm{a}$ and Figures S4.1 and S4.2).

Stability. The ultimate goal of exploiting excited state aromaticity to generate singlet fission-capable materials is to access triplet states without cost to the chemical stability. The 

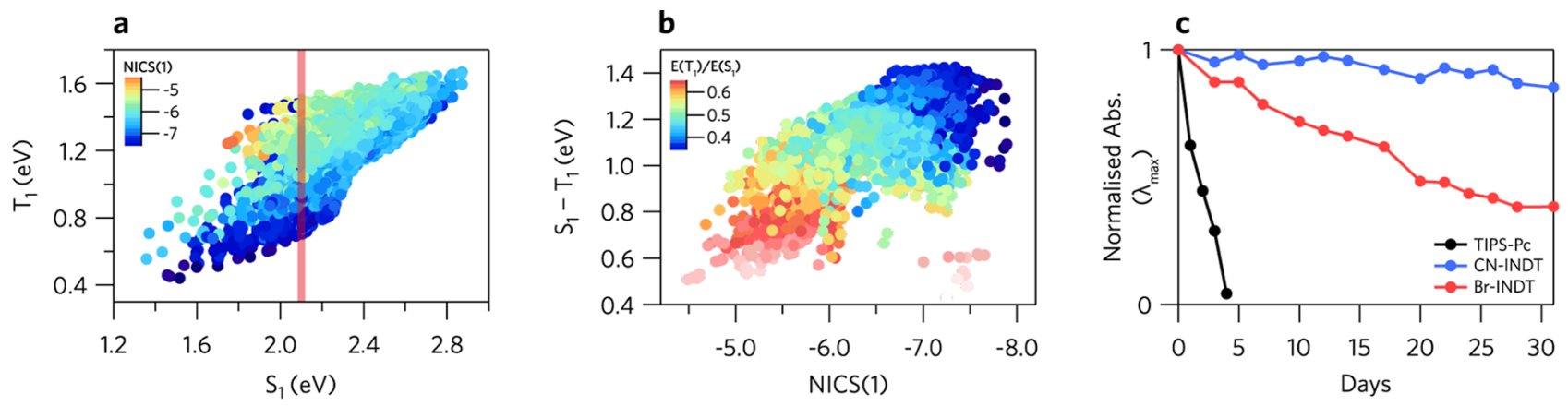

Figure 5. Ambient stability and excited state aromaticity of INDT compounds. (a) Calculated energies of $S_{1}$ and $T_{1}$ for 9920 candidates with the corresponding C5 NICS(1) shown in the color map. Red bar shows $S_{1}=2.1 \mathrm{eV}$. (b) The relationship between the $S-\mathrm{T}$ gap and triplet state aromaticity with the corresponding $T_{1} / S_{1}$ ratio shown in color map. (c) Normalized intensity of the $\lambda_{\max }$ of the steady-state absorbance spectra of thin-films of TIPS-pentacene (TIPS-Pc), Br-INDT and CN-INDT spun from $5 \mathrm{mg} / \mathrm{mL}$ solutions in chloroform over 31 days. Spectra of all materials are shown in the SI Section S6.

ambient stability of each INDT material was compared to TIPS-pentacene. Thin films were identically prepared and stored in individual clear Perspex boxes on a shelf, in air, in a well-lit area, but out of direct sunlight, and the steady state absorption was measured periodically over 31 days (see SI Section S6). Under these ambient conditions TIPS-pentacene showed total degradation after 4 days, while the INDT materials exhibited excellent ambient stability and CN-INDT maintained over $80 \%$ of its original absorbance after 31 days (Figure 5c). The high chemical stability of the INDTs showcases their robust ground and excited states despite fission occurring readily (and hence a high concentration of triplets being present) in the material. By exploiting triplet aromaticity, not only is the energy of the triplet state lowered such that it can undergo singlet fission, but the delocalized nature of the unpaired electrons means they are less susceptible to photodegradation reactions, in an analogous way that ground state aromaticity increases the stability of organic compounds. Triplet-mediated photodegradation of acenes and other polyaromatic hydrocarbons is welldocumented, thus introducing some aromatic character into excited states could lead to a generalized method to enhance the photostability of organic materials.

We note that the increase in triplet-state aromaticity does come at some slight cost to ground-state aromaticity, but this effect is negligible as the materials also possess significant ground state aromaticity as evidenced by their high stability in the ground state as well.

Expanding the Chemical Space. The experimental proof of singlet fission in INDT-based materials and their photochemical stability benchmarks the essential validity of our approach: increasing triplet aromaticity through chemical functionalization results in more energetically favorable singlet fission, into more stable triplet states. We now return to our design principle to explore the scope of materials tunability and identify robust trends linking excited-state energetics and triplet aromaticity. To widen our analysis, theoretical energies of $S_{1}$ and $T_{1}$ and NICS(1) aromaticity for 9920 indolonaphthyridine candidates were calculated using TD-DFT incorporating the Tamm-Dancoff approximation (TDA) at the B3LYP/6-311++G** level of theory (rationale of these methods given in the SI Section S7). The results and codes used to generate and perform all calculations detailed in this report are provided online in a publicly available repository (https://github.com/SMTG-UCL/singlet-fission-screening).
In this wider sample group (Figure 5a), the correlation of increased excited state aromaticity and reduced $T_{1} / S_{1}$ is unequivocally clear. It demonstrates that, through appropriate chemical substitution to increase the aromaticity of the triplet state, its energy can be lowered without affecting the energy of the singlet-the red bar highlights candidates with $S_{1}=2.1 \mathrm{eV}$ and with $\mathrm{T}_{1}$ ranging from $1.5-0.5 \mathrm{eV}$. We can directly visualize this tuning by plotting the calculated $S_{1}-T_{1}$ energy gap as a function of triplet-state aromaticity (Figure $5 \mathrm{~b}$ ). These results directly demonstrate that exploiting Baird-type aromaticity is a powerful approach for manipulating the singlet-triplet energy gap. Independent manipulation of the singlet and triplet energies in such a manner has never been demonstrated and has implications across many areas of science such as light emitting diodes, photovoltaics, photocatalysis, photochemistry, spintronics, and magnetism. In the context of singlet fission, this data has allowed us to identify 2616 new singlet fission candidates with a wide range of optical band-gaps and $\mathrm{T} / \mathrm{S}$ ratios. These are all based on straightforward substitution of the indolonaphthyridine chromophore, which is itself wellknown for photochemical robustness and large oscillator strength-both ideal properties for applications of singlet fission.

Applicability to Other Systems. In order to demonstrate the application and generalizability of the proposed methodology we demonstrate here that excited state aromaticity can be used to rationalize the energetic position of alternative nonacene singlet fission candidates and also as a predictive tool to search for new materials which fulfill the energetic design criteria. Diketopyrolopyrole (DPP) has been suggested to undergo singlet fission but thus far there has been no explanation for its unusually large $S_{1}-T_{1}$ energy gap. ${ }^{66}$ Similarly to our Cibalackrot derivatives, we propose that the presence of $[4 n]$ resonance structures induces aromaticity of the triplet excited state, resulting in a widening of the $S_{1}-T_{1}$ energy gap such that fission becomes energetically feasible.

Figure 6 shows amide bond resonances which result in a core which has an electronic structure similar to pentalene, which has an aromatic triplet state. ${ }^{42}$ This aromaticity of the triplet state of DPP is confirmed by NICS calculations (each central-ring has NICS(1) -3.96 using DFT/TDDFT B3LYP/ $\left.6-311 \mathrm{G}^{* *}\right)$. As detailed earlier, we suggest this is responsible for its energetic stabilization and potentially also explains the superior stability toward photodegradation of this chromo- 
<smiles>CN1C(=O)C2=C(c3cccs3)N(C)C(=O)C2=C1c1cccs1</smiles><smiles>C[N+]1=C(O)C2=C([O-])[N+](C)=C(c3cccs3)C2=C1c1cccs1</smiles>
c.f.
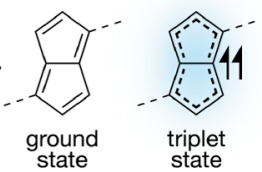

Figure 6. Amide resonances of diketopyrrolopyrrole (DPP) and its similarity to pentalene, which has an aromatic triplet excited state.

phore (the unpaired electrons are delocalized rendering them less reactive to photodegradation reactions).

Implementation of the proposed design rules to identify and design new chromophores as singlet fission candidates or merely as a tool to predict and rationalize the relative excited state energetics is demonstrated below. Table 1 shows the structures and calculated vertical excited state energies (using DFT/TDDFT B3LYP/6-311G**) of two sets of molecules A and $\mathbf{B}$ and their two regioisomers ( 5 or $\mathbf{6}$ ) based on the size (and connectivity) of the lactam ring. It can clearly be seen that within each set of isomers (i.e., A5 vs A6) virtually all parameters that could affect excited state energies (e.g., torsion angles, conjugation paths, atom connectivity) are identical with the exception of the core ring size.

It should be noted that all calculations are performed at the ground state geometry where the amide-containing cores are completely planar. Similarly to INDT and DPP, in both cases where a 5-membered cyclic amide is present (A5 and B5) it is possible to draw resonance structures (see Figure S2.5) that contain $[4 n]$ electrons suggesting that the triplet excited state will have aromatic character. In both cases where the central core is comprised of 6-membered rings (A6 and B6) the resulting resonance structures have $[4 n+2]$ electrons, suggesting that the excited states will have antiaromatic character. Therefore, one can simply predict without even the aid of theoretical calculations that the compounds with 5membered cyclic amides will have narrower optical energy gaps, and larger $S_{1}-T_{1}$ energy gaps, making them singlet fission candidates. Both of these predictions, alongside confirmation of the reversal of aromaticity are completely supported by the theoretical calculations (Table 1).

It is important to note that although these dyes have been previously reported, ${ }^{67,68}$ neither A5 nor B5 have been investigated for singlet fission, indicating the ease with which new candidates that fulfill the energetic criteria for singlet fission can be identified using this theory. Additionally, the ease with which $[4 n]$ vs $[4 n+2]$ cyclic motifs can be identified adds an extremely powerful tool for the synthetic chemist to predict and rationalize optical energy gaps.

The inclusion of some ground-state antiaromaticity need not compromise stability of these chromophores as all the materials presented here have substantial overall aromatic character, and experimentally both DPP and Cibalackrot are extremely stable chromophores. As seen in Figure 4, the magnitude of the $S_{1}-T_{1}$ energy gap can be tuned and we suggest that in order to control this value through chemical design it is necessary to investigate the relative contributions of the excited states (both $S_{1}$ and $T_{1}$ ) to the aromatic $[4 \mathrm{n}]$ core as Baird's rules formally applies to both types of excited states, which is beyond the scope of this investigation. Additionally, we again note that our calculations are performed at a relatively low level of theory, but the predicted values of the compounds presented in Table 1 are in good agreement with experiment. $^{67,68}$ Finally, we note that solid-state intermolecular interactions play a very significant role in the process of the singlet fission. ${ }^{12,27,51,69,70}$ These are not considered in our theoretical analysis of new core chromophores, but where the intermolecular coupling motifs are known it should be possible to optimize the geometry either experimentally ${ }^{12,27,70}$ or computationally. $51,69,71$

\section{CONCLUSIONS}

Though our experimental exploration has been limited to a single family of materials, the wide tunability we have demonstrated, our preliminary calculations on alternative [4n] containing dyes, and previous investigations of Bairdtype aromaticity ${ }^{41-43,72}$ indicate that the same principles can be readily applied to other materials systems. In practical terms, the requirement of a $[4 n]$ cyclic contribution to a molecule's resonance structures translates into a simple design rule for targeting singlet fission systems. Examples of these (beyond INDT) are structures such as DPP (which has been proposed to undergo singlet fission, but no rationale for its large singlet-triplet energy gap has been suggested), indigo, isoindigo, ${ }^{73}$ pechmann dyes, and fulvalenes. Ground state aromaticity is a fundamental cornerstone of synthetic organic chemistry, providing stability and tunability to unsaturated systems, underpinning the entire development of the field of organic electronics. The work we present here demonstrates that excited-state aromaticity plays an equally important role in both the energetic position and the stability of photoexcited

Table 1. Structures and Theoretical Excited State Properties of Two Molecules A and B, and Their Two Isomers 5 (Bridged C5 Lactam Rings) and 6 (Fused C6 Lactam Rings) Calculated at the TDDFT/B3LYP 6-311G* Level of Theory
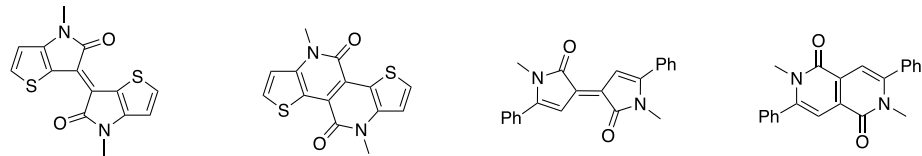

\begin{tabular}{c|cc|cc}
\hline Molecule & A5 & A6 & B5 & B6 \\
\hline $\mathrm{S}_{1}(\mathrm{eV})$ & 2.29 & 3.11 & 2.20 & 3.22 \\
$\mathrm{~T}_{1}(\mathrm{eV})$ & 1.04 & 1.94 & 0.73 & 0.11 \\
$\mathrm{~T}_{1} / \mathrm{S}_{1}$ & 0.45 & 0.62 & 0.33 & 0.66 \\
$\mathrm{~S}_{1}-\mathrm{T}_{1}(\mathrm{eV})$ & 1.25 & 1.17 & 1.47 & 1.10 \\
Triplet NICS(1) & -3.1 & +5.2 & -3.4 & +8.7 \\
\hline
\end{tabular}


triplet states. More broadly, we envisage the same approach may be used to tune the exchange energy in organic systems where triplets play a critical role, to mitigate losses in photovoltaics, harvest additional carriers in light-emitting diodes, enhance the stability of novel open-shell systems, or broaden the materials library for photon up-conversion and singlet fission.

\section{ASSOCIATED CONTENT}

\section{S Supporting Information}

The Supporting Information is available free of charge on the ACS Publications website at DOI: 10.1021/jacs.9b06346.

Synthetic methods and characterization, spectroscopic data and analysis (PDF)

\section{AUTHOR INFORMATION}

\section{Corresponding Authors}

*rhf10@cam.ac.uk

*ajm557@cornell.edu

*hab60@cam.ac.uk

\section{ORCID}

Kealan J. Fallon: 0000-0001-6241-6034

Christopher N. Savory: 0000-0002-9052-7484

Chad Risko: 0000-0001-9838-5233

David O. Scanlon: 0000-0001-9174-8601

Christopher W. M. Kay: 0000-0002-5200-6004

Akshay Rao: 0000-0003-4261-0766

Richard H. Friend: 0000-0001-6565-6308

Andrew J. Musser: 0000-0002-4600-6606

Hugo Bronstein: 0000-0003-0293-8775

\section{Present Address}

${ }^{\infty}$ Department of Chemistry \& Chemical Biology, Cornell University, Ithaca, New York 14853, United States.

\section{Author Contributions}

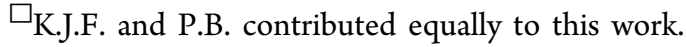

\section{Notes}

The authors declare no competing financial interest.

Computational data is available in a publicly available repository at https://github.com/SMTG-UCL/singlet-fissionscreening.

\section{ACKNOWLEDGMENTS}

Part of this work was funded by EU project 679789 CONTREX and EPSRC grant "Centre for Advanced Materials for Integrated Energy Systems (CAM-IES)” EP/P007767/1. A.J.M. was supported by the EPSRC (EP/M01083X/1 and $\mathrm{EP} / \mathrm{M} 025330 / 1)$. This work made use of the ARCHER UK National Supercomputing Service (http://www.archer.ac.uk) via our membership of the UK's HEC Materials Chemistry Consortium, which is funded by EPSRC (EP/L000202), and the Thomas machine which is funded by the Tier $2 \mathrm{Hub}$ in Materials and Molecular Modelling (EP/P020194/1). A.M.G. acknowledges Diamond Light Source for the cosponsorship of a studentship on the EPSRC Centre for Doctoral Training in Molecular Modelling and Materials Science (EP/L015862/1). C.N.S. is grateful to the EPSRC and the Department of Chemistry at UCL for the provision of a Doctoral Training Partnership studentship (Ref No. 1492829).

\section{REFERENCES}

(1) Congreve, D. N.; Lee, J.; Thompson, N. J.; Hontz, E.; Yost, S. R.; Reusswig, P. D.; Bahlke, M. E.; Reineke, S.; Van Voorhis, T.; Baldo, M. A. External Quantum Efficiency above $100 \%$ in a Singlet-ExcitonFission-Based Organic Photovoltaic Cell. Science 2013, 340 (6130), 334-337.

(2) Walker, B. J.; Musser, A. J.; Beljonne, D.; Friend, R. H. Singlet Exciton Fission in Solution. Nat. Chem. 2013, 5 (12), 1019-1024.

(3) Sanders, S. N.; Kumarasamy, E.; Pun, A. B.; Trinh, M. T.; Choi, B.; Xia, J.; Taffet, E. J.; Low, J. Z.; Miller, J. R.; Roy, X.; et al. Quantitative Intramolecular Singlet Fission in Bipentacenes. J. Am. Chem. Soc. 2015, 137 (28), 8965-8972.

(4) Johnson, J. C.; Nozik, A. J.; Michl, J. High Triplet Yield from Singlet Fission in a Thin Film of 1,3-Diphenylisobenzofuran. J. Am. Chem. Soc. 2010, 132, 16302-16303.

(5) Burdett, J. J.; Muller, A. M.; Gosztola, D.; Bardeen, C. J. Excited State Dynamics in Solid and Monomeric Tetracene: The Roles of Superradiance and Exciton Fission. J. Chem. Phys. 2010, 133 (14), 144506.

(6) Hanna, M. C.; Nozik, A. J. Solar Conversion Efficiency of Photovoltaic and Photoelectrolysis Cells with Carrier Multiplication Absorbers. J. Appl. Phys. 2006, 100 (7), 074510.

(7) Shockley, W.; Queisser, H. J. Detailed Balance Limit of Efficiency of P-n Junction Solar Cells. J. Appl. Phys. 1961, 32 (3), 510-519.

(8) Rao, A.; Friend, R. H. Harnessing Singlet Exciton Fission to Break the Shockley-Queisser Limit. Nat. Rev. Mater. 2017, 2, 17063.

(9) Eaton, S. W.; Miller, S. A.; Margulies, E. A.; Shoer, L. E.; Schaller, R. D.; Wasielewski, M. R. Singlet Exciton Fission in Thin Films of Tert -Butyl-Substituted Terrylenes. J. Phys. Chem. A 2015, 119 (18), 4151-4161.

(10) Margulies, E. A.; Miller, C. E.; Wu, Y.; Ma, L.; Schatz, G. C.; Young, R. M.; Wasielewski, M. R. Enabling Singlet Fission by Controlling Intramolecular Charge Transfer in $\pi$-Stacked Covalent Terrylenediimide Dimers. Nat. Chem. 2016, 8 (12), 1120-1125.

(11) Le, A. K.; Bender, J. A.; Roberts, S. T. Slow Singlet Fission Observed in a Polycrystalline Perylenediimide Thin Film. J. Phys. Chem. Lett. 2016, 7 (23), 4922.

(12) Le, A. K.; Bender, J. A.; Arias, D. H.; Cotton, D. E.; Johnson, J. C.; Roberts, S. T. Singlet Fission Involves an Interplay between Energetic Driving Force and Electronic Coupling in Perylenediimide Films. J. Am. Chem. Soc. 2018, 140 (2), 814-826.

(13) Wang, C.; Tauber, M. J. High-Yield Singlet Fission in a Zeaxanthin Aggregate Observed by Picosecond Resonance Raman Spectroscopy. J. Am. Chem. Soc. 2010, 132 (40), 13988-13991.

(14) Musser, A. J.; Al-Hashimi, M.; Maiuri, M.; Brida, D.; Heeney, M.; Cerullo, G.; Friend, R. H.; Clark, J. Activated Singlet Exciton Fission in a Semiconducting Polymer. J. Am. Chem. Soc. 2013, 135 (34), 12747-12754.

(15) Musser, A. J.; Maiuri, M.; Brida, D.; Cerullo, G.; Friend, R. H.; Clark, J. The Nature of Singlet Exciton Fission in Carotenoid Aggregates. J. Am. Chem. Soc. 2015, 137 (15), 5130-5139.

(16) Busby, E.; Xia, J.; Wu, Q.; Low, J. Z.; Song, R.; Miller, J. R.; Zhu, X.-Y.; Campos, L. M.; Sfeir, M. Y. A Design Strategy for Intramolecular Singlet Fission Mediated by Charge-Transfer States in Donor-Acceptor Organic Materials. Nat. Mater. 2015, 14 (4), 426433.

(17) Kasai, Y.; Tamai, Y.; Ohkita, H.; Benten, H.; Ito, S. Ultrafast Singlet Fission in a Push-Pull Low-Bandgap Polymer Film. J. Am. Chem. Soc. 2015, 137 (51), 15980-15983.

(18) Huynh, U. N. V.; Basel, T. P.; Ehrenfreund, E.; Li, G.; Yang, Y.; Mazumdar, S.; Vardeny, Z. V. Transient Magnetophotoinduced Absorption Studies of Photoexcitations in Pi-Conjugated DonorAcceptor Copolymers. Phys. Rev. Lett. 2017, 119 (1), 017401.

(19) Wilson, M. W. B.; Rao, A.; Clark, J.; Kumar, R. S. S.; Brida, D.; Cerullo, G.; Friend, R. H. Ultrafast Dynamics of Exciton Fission in Polycrystalline Pentacene. J. Am. Chem. Soc. 2011, 133 (31), 1183011833. 
(20) Miyata, K.; Kurashige, Y.; Watanabe, K.; Sugimoto, T.; Takahashi, S.; Tanaka, S.; Takeya, J.; Yanai, T.; Matsumoto, Y. Coherent Singlet Fission Activated by Symmetry Breaking. Nat. Chem. 2017, 9 (10), 983-989.

(21) Grieco, C.; Kennehan, E. R.; Kim, H.; Pensack, R. D.; Brigeman, A. N.; Rimshaw, A.; Payne, M. M.; Anthony, J. E.; Giebink, N. C.; Scholes, G. D.; et al. Direct Observation of Correlated Triplet Pair Dynamics during Singlet Fission Using Ultrafast Mid-IR Spectroscopy. J. Phys. Chem. C 2018, 122 (4), 2012-2022.

(22) Folie, B. D.; Haber, J. B.; Refaely-Abramson, S.; Neaton, J. B.; Ginsberg, N. S. Long-Lived Correlated Triplet Pairs in a $\pi$-Stacked Crystalline Pentacene Derivative. J. Am. Chem. Soc. 2018, 140 (6), 2326-2335.

(23) Stern, H. L.; Cheminal, A.; Yost, S. R.; Broch, K.; Bayliss, S. L.; Chen, K.; Tabachnyk, M.; Thorley, K.; Greenham, N.; Hodgkiss, J. M.; et al. Vibronically Coherent Ultrafast Triplet-Pair Formation and Subsequent Thermally Activated Dissociation Control Efficient Endothermic Singlet Fission. Nat. Chem. 2017, 9 (12), 1205-1212.

(24) Roberts, S. T.; McAnally, R. E.; Mastron, J. N.; Webber, D. H.; Whited, M. T.; Brutchey, R. L.; Thompson, M. E.; Bradforth, S. E. Efficient Singlet Fission Discovered in a Disordered Acene Film. J. Am. Chem. Soc. 2012, 134 (14), 6388-6400.

(25) Burdett, J. J.; Bardeen, C. J. The Dynamics of Singlet Fission in Crystalline Tetracene and Covalent Analogs. Acc. Chem. Res. 2013, 46 (6), 1312-1320.

(26) Tayebjee, M. J.; Clady, R. G.; Schmidt, T. W. The Exciton Dynamics in Tetracene Thin Films. Phys. Chem. Chem. Phys. 2013, 15 (35), 14797-14805.

(27) Yost, S. R.; Lee, J.; Wilson, M. W. B.; Wu, T.; McMahon, D. P.; Parkhurst, R. R.; Thompson, N. J.; Congreve, D. N.; Rao, A.; Johnson, K.; et al. A Transferable Model for Singlet-Fission Kinetics. Nat. Chem. 2014, 6 (6), 492-497.

(28) Musser, A. J.; Liebel, M.; Schnedermann, C.; Wende, T.; Kehoe, T. B.; Rao, A.; Kukura, P. Evidence for Conical Intersection Dynamics Mediating Ultrafast Singlet Exciton Fission. Nat. Phys. 2015, 11 (4), 352-357.

(29) Herz, J.; Buckup, T.; Paulus, F.; Engelhart, J. U.; Bunz, U. H. F.; Motzkus, M. Unveiling Singlet Fission Mediating States in TIPSPentacene and Its Aza Derivatives. J. Phys. Chem. A 2015, 119 (25), 6602-6610.

(30) Pensack, R. D.; Tilley, A. J.; Parkin, S. R.; Lee, T. S.; Payne, M. M.; Gao, D.; Jahnke, A. A.; Oblinsky, D. G.; Li, P. F.; Anthony, J. E.; et al. Exciton Delocalization Drives Rapid Singlet Fission in Nanoparticles of Acene Derivatives. J. Am. Chem. Soc. 2015, 137 (21), 6790-6803.

(31) Bakulin, A. A.; Morgan, S. E.; Kehoe, T. B.; Wilson, M. W. B.; Chin, A. W.; Zigmantas, D.; Egorova, D.; Rao, A. Real-Time Observation of Multiexcitonic States in Ultrafast Singlet Fission Using Coherent 2D Electronic Spectroscopy. Nat. Chem. 2016, 8 (1), 1623.

(32) Northrop, B. H.; Houk, K. N.; Maliakal, A. Photostability of Pentacene and 6,13-Disubstituted Pentacene Derivatives: A Theoretical and Experimental Mechanistic Study. Photochem. Photobiol. Sci. 2008, 7 (12), 1463-1468.

(33) Zade, S. S.; Zamoshchik, N.; Reddy, A. R.; Fridman-Marueli, G.; Sheberla, D.; Bendikov, M. Products and Mechanism of Acene Dimerization. A Computational Study. J. Am. Chem. Soc. 2011, 133 (28), 10803-10816.

(34) Paci, I.; Johnson, J. C.; Chen, X.; Rana, G.; Popović, D.; David, D. E.; Nozik, A. J.; Ratner, M. A.; Michl, J. Singlet Fission for DyeSensitized Solar Cells: Can a Suitable Sensitizer Be Found? J. Am. Chem. Soc. 2006, 128 (51), 16546-16553.

(35) Minami, T.; Nakano, M. Diradical Character View of Singlet Fission. J. Phys. Chem. Lett. 2012, 3 (2), 145-150.

(36) Wen, J.; Havlas, Z.; Michl, J. Captodatively Stabilized Biradicaloids as Chromophores for Singlet Fission. J. Am. Chem. Soc. 2015, 137 (1), 165-172.

(37) Lukman, S.; Richter, J. M.; Yang, L.; Hu, P.; Wu, J.; Greenham, N. C.; Musser, A. J. Efficient Singlet Fission and Triplet-Pair Emission in a Family of Zethrene Diradicaloids. J. Am. Chem. Soc. 2017, 139 (50), 18376.

(38) Hu, P.; Wu, J. Modern Zethrene Chemistry. Can. J. Chem. 2017, 95 (3), 223-233.

(39) Baird, N. C. Quantum Organic Photochemistry. II. Resonance and Aromaticity in the Lowest $3 \pi \pi^{*}$ State of Cyclic Hydrocarbons. J. Am. Chem. Soc. 1972, 94 (14), 4941-4948.

(40) Ottosson, H. Exciting Excited-State Aromaticity. Nat. Chem. 2012, 4, 969.

(41) Möllerstedt, H.; Piqueras, M. C.; Crespo, R.; Ottosson, H. Fulvenes, Fulvalenes, and Azulene: Are They Aromatic Chameleons? J. Am. Chem. Soc. 2004, 126 (43), 13938-13939.

(42) Ayub, R.; Bakouri, O. El; Jorner, K.; Solà, M.; Ottosson, H. Can Baird's and Clar's Rules Combined Explain Triplet State Energies of Polycyclic Conjugated Hydrocarbons with Fused $4 n \pi$ - and $(4 n+2) \pi$ Rings? J. Org. Chem. 2017, 82 (12), 6327-6340.

(43) Villaume, S.; Fogarty, H. A.; Ottosson, H. Triplet-State Aromaticity of $4 \mathrm{n} \pi$-Electron Monocycles: Analysis of Bifurcation in the $\pi$ Contribution to the Electron Localization Function. ChemPhysChem 2008, 9 (2), 257-264.

(44) Oh, J.; Sung, Y. M.; Mori, H.; Park, S.; Jorner, K.; Ottosson, H.; Lim, M.; Osuka, A.; Kim, D. Unraveling Excited-Singlet-State Aromaticity via Vibrational Analysis. Chem. 2017, 3 (5), 870-880.

(45) Ueda, M.; Jorner, K.; Sung, Y. M.; Mori, T.; Xiao, Q.; Kim, D.; Ottosson, H.; Aida, T.; Itoh, Y. Energetics of Baird Aromaticity Supported by Inversion of Photoexcited Chiral [4n]Annulene Derivatives. Nat. Commun. 2017, 8 (1), 346.

(46) Cha, W.-Y.; Kim, T.; Ghosh, A.; Zhang, Z.; Ke, X.-S.; Ali, R.; Lynch, V. M.; Jung, J.; Kim, W.; Lee, S.; et al. Bicyclic Baird-Type Aromaticity. Nat. Chem. 2017, 9, 1243.

(47) Hada, M.; Saito, S.; Tanaka, S.; Sato, R.; Yoshimura, M.; Mouri, K.; Matsuo, K.; Yamaguchi, S.; Hara, M.; Hayashi, Y.; et al. Structural Monitoring of the Onset of Excited-State Aromaticity in a Liquid Crystal Phase. J. Am. Chem. Soc. 2017, 139 (44), 15792-15800.

(48) Engi, G. Über Neue Derivate Des Indigos Und Anderer Indigoider Farbstoffe. Angew. Chem. 1914, 27 (20), 144-148.

(49) Smith, M. B.; Michl, J. Recent Advances in Singlet Fission. Annu. Rev. Phys. Chem. 2013, 64, 361-386.

(50) Smith, M.; Michl, J. Singlet Fission. Chem. Rev. 2010, 110 (6), 6891-6936.

(51) Buchanan, E. A.; Havlas, Z.; Michl, J. Singlet Fission: Optimization of Chromophore Dimer Geometry. Adv. Quantum Chem. 2017, 75, 175-227.

(52) Peach, M. J. G.; Williamson, M. J.; Tozer, D. J. Influence of Triplet Instabilities in TDDFT. J. Chem. Theory Comput. 2011, 7 (11), $3578-3585$.

(53) Geuenich, D.; Hess, K.; Köhler, F.; Herges, R. Anisotropy of the Induced Current Density (ACID), a General Method To Quantify and Visualize Electronic Delocalization. Chem. Rev. 2005, 105 (10), 3758-3772.

(54) Herges, R.; Geuenich, D. Delocalization of Electrons in Molecules. J. Phys. Chem. A 2001, 105 (13), 3214-3220.

(55) Spano, F. C. The Spectral Signatures of Frenkel Polarons in Hand J-Aggregates. Acc. Chem. Res. 2010, 43 (3), 429-439.

(56) Eaton, S. W.; Shoer, L. E.; Karlen, S. D.; Dyar, S. M.; Margulies, E. A.; Veldkamp, B. S.; Ramanan, C.; Hartzler, D. A.; Savikhin, S.; Marks, T. J.; et al. Singlet Exciton Fission in Polycrystalline Thin Films of a Slip-Stacked Perylenediimide. J. Am. Chem. Soc. 2013, 135 (39), 14701-14712.

(57) Albert-Seifried, S.; Friend, R. H. Measurement of Thermal Modulation of Optical Absorption in Pump-Probe Spectroscopy of Semiconducting Polymers. Appl. Phys. Lett. 2011, 98 (22), 223304.

(58) Rao, A.; Wilson, M. W. B.; Albert-Seifried, S.; Di Pietro, R.; Friend, R. H. Photophysics of Pentacene Thin Films: The Role of Exciton Fission and Heating Effects. Phys. Rev. B: Condens. Matter Mater. Phys. 2011, 84 (19), 195411.

(59) Weiss, L. R.; Bayliss, S. L.; Kraffert, F.; Thorley, K. J.; Anthony, J. E.; Bittl, R.; Friend, R. H.; Rao, A.; Greenham, N. C.; Behrends, J. 
Strongly Exchange-Coupled Triplet Pairs in an Organic Semiconductor. Nat. Phys. 2017, 13 (2), 176-181.

(60) Tayebjee, M. J. Y.; Sanders, S. N.; Kumarasamy, E.; Campos, L. M.; Sfeir, M. Y.; McCamey, D. R. Quintet Multiexciton Dynamics in Singlet Fission. Nat. Phys. 2017, 13 (2), 182-188.

(61) Basel, B. S.; Zirzlmeier, J.; Hetzer, C.; Phelan, B. T.; Krzyaniak, M. D.; Reddy, S. R.; Coto, P. B.; Horwitz, N. E.; Young, R. M.; White, F. J.; et al. Unified Model for Singlet Fission within a Non-Conjugated Covalent Pentacene Dimer. Nat. Commun. 2017, 8, 1-8.

(62) Kumarasamy, E.; Sanders, S. N.; Tayebjee, M. J. Y.; Asadpoordarvish, A.; Hele, T. J. H.; Fuemmeler, E. G.; Pun, A. B.; Yablon, L. M.; Low, J. Z.; Paley, D. W.; et al. Tuning Singlet Fission in $\pi$-Bridge- $\pi$ Chromophores. J. Am. Chem. Soc. 2017, 139 (36), 1248812494 .

(63) Lubert-Perquel, D.; Salvadori, E.; Dyson, M.; Stavrinou, P. N.; Montis, R.; Nagashima, H.; Kobori, Y.; Heutz, S.; Kay, C. W. M. Identifying Triplet Pathways in Dilute Pentacene Films. Nat. Commun. 2018, 9 (1), 4222.

(64) Chen, K.; Gallaher, J. K.; Barker, A. J.; Hodgkiss, J. M. Transient Grating Photoluminescence Spectroscopy: An Ultrafast Method of Gating Broadband Spectra. J. Phys. Chem. Lett. 2014, 5 (10), 1732-1737.

(65) Mou, W.; Hattori, S.; Rajak, P.; Shimojo, F.; Nakano, A. Nanoscopic Mechanisms of Singlet Fission in Amorphous Molecular Solid. Appl. Phys. Lett. 2013, 102 (17), 173301.

(66) Mauck, C. M.; Hartnett, P. E.; Margulies, E. A.; Ma, L.; Miller, C. E.; Schatz, G. C.; Marks, T. J.; Wasielewski, M. R. Singlet Fission via an Excimer-Like Intermediate in 3,6-Bis(Thiophen-2-Yl)Diketopyrrolopyrrole Derivatives. J. Am. Chem. Soc. 2016, 138, 11749.

(67) Ashraf, R. S.; Kronemeijer, A. J.; James, D. I.; Sirringhaus, H.; McCulloch, I. A New Thiophene Substituted Isoindigo Based Copolymer for High Performance Ambipolar Transistors. Chem. Commun. 2012, 48 (33), 3939-3941.

(68) Kantchev, E. A. B.; Norsten, T. B.; Sullivan, M. B. TimeDependent Density Functional Theory (TDDFT) Modelling of Pechmann Dyes: From Accurate Absorption Maximum Prediction to Virtual Dye Screening. Org. Biomol. Chem. 2012, 10 (33), 66826692.

(69) Renaud, N.; Sherratt, P. A.; Ratner, M. A. Mapping the Relation between Stacking Geometries and Singlet Fission Yield in a Class of Organic Crystals. J. Phys. Chem. Lett. 2013, 4 (7), 1065-1069.

(70) Schrauben, J. N.; Ryerson, J. L.; Michl, J.; Johnson, J. C. Mechanism of Singlet Fission in Thin Films of 1,3-Diphenylisobenzofuran. J. Am. Chem. Soc. 2014, 136 (20), 7363-7373.

(71) Greyson, E. C.; Stepp, B. R.; Chen, X.; Schwerin, A. F.; Paci, I.; Smith, M. B.; Akdag, A.; Johnson, J. C.; Nozik, A. J.; Michl, J.; et al. Singlet Exciton Fission for Solar Cell Applications: Energy Aspects of Interchromophore Coupling ${ }^{\dagger}$. J. Phys. Chem. B 2010, 114 (45), 14223-14232.

(72) Sung, Y. M.; Yoon, M. C.; Lim, J. M.; Rath, H.; Naoda, K.; Osuka, A.; Kim, D. Reversal of Hückel (Anti)Aromaticity in the Lowest Triplet States of Hexaphyrins and Spectroscopic Evidence for Baird's Rule. Nat. Chem. 2015, 7 (5), 418-422.

(73) Hu, J.; Xu, K.; Shen, L.; Wu, Q.; He, G.; Wang, J.-Y.; Pei, J.; Xia, J.; Sfeir, M. Y. New Insights into the Design of Conjugated Polymers for Intramolecular Singlet Fission. Nat. Commun. 2018, 9 (1), 2999. 